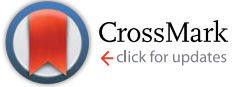

Cite this: RSC Adv., 2017, 7, 6209

\title{
SDS induced dissociation of STY3178 oligomer: experimental and molecular dynamics studies
}

\author{
Paramita Saha, ${ }^{a}$ Samapan Sikdar, ${ }^{a}$ Camelia Manna, ${ }^{a}$ Jaydeb Chakrabarti ${ }^{\mathrm{ab}}$ \\ and Mahua Ghosh*a
}

STY3178 is a yfdX protein from Salmonella Typhi. yfdX proteins occur ubiquitously in a number of virulent bacteria but their cellular localization is unknown. Our earlier studies have shown that STY3178 is a trimer and can be a periplasmic chaperone protein. In the present study we show the stability of STY3178 in the presence of the bio-mimetic anionic surfactant sodium dodecyl sulphate (SDS). With increasing concentrations of SDS we observe monomeric STY3178 which reversibly forms the trimer upon decreasing the surfactant concentration. Protein tertiary structure is not perturbed in the presence of SDS. We show using molecular dynamics simulation and conformational thermodynamics data that SDS induces stability of the monomer compared to an isolated monomer of STY3178. This supports our experimental observations.

Received 24th October 2016 Accepted 3rd January 2017

DOI: 10.1039/c6ra25737b

www.rsc.org/advances

\section{Introduction}

Surfactants mimic cellular membrane properties and hence find application in the isolation and solubilization of proteins. ${ }^{1,2}$ In particular membrane proteins are often solubilized in surfactants in the absence of lipids. These surfactants sometimes affect protein tertiary or quaternary structure. Ionic surfactants are amphiphilic in nature and show electrostatic interaction with proteins through ionic head groups and hydrophobic interactions using the hydrocarbon tail, whereas the non-ionic surfactants interact only via hydrophobic interactions. ${ }^{3}$ Protein interaction with surfactants finds applications in many different industries like the chemical, pharmaceutical including drug delivery, food and cosmetic industries. ${ }^{4-6}$

Study of interaction of surfactants with bacterial proteins might provide vital information regarding the host cell surface interactions. It can also provide clue to the intracellular interactions between periplasmic protein and cellular membrane. yfdX proteins are ubiquitously present in virulent bacteria. However, the exact cellular localization for these proteins is still unknown. Importance of these proteins are indicated by the following: (i) its presence in disease causing bacteria, (ii) its upregulation in $E$. coli by a multidrug response regulator protein Evg $^{7}$ and (iii) its interaction with antibiotics like ciprofloxacin, rifampin and ampicillin for Salmonella Typhi protein STY3178. ${ }^{7}$ It is also reported that STY3178 show stability in wide range of

${ }^{a}$ Department of Chemical, Biological and Macromolecular Sciences, S. N. Bose National Centre for Basic Sciences, Block JD, Sector III, Salt lake, Kolkata 700106, India.E-mail: mahuaghosh@bose.res.in; mahua.ghosh@gmail.com

${ }^{b}$ The Thematic Unit of Excellence on Computational Materials Science, S. N. Bose National Centre for Basic Sciences, Block JD, Sector III, Salt lake, Kolkata 700106, India
pH. ${ }^{8}$ We have shown the thermally stable STY3178 has the chaperone activity capability in absence of ATP like known periplasmic chaperones. ${ }^{9}$ STY3178 shows reversibility in unfolding in presence of thermal ${ }^{9}$ and chemical perturbations. ${ }^{8}$

Here we study the effect of most widely used anionic surfactant sodium dodecyl sulphate (SDS) on the structural stability of STY3178. SDS is known to affect protein structure in a variety of ways. For instance, $\alpha$-helical and $\alpha / \beta$ mix proteins appear to be more prone to SDS-induced unfolding than the $\beta$ sheeted ones. ${ }^{3}$ Above the critical micellar concentration (CMC), SDS stabilizes $\alpha$-helical conformation, ${ }^{\mathbf{1 0 - 1 2}}$ whereas below CMC, it stabilizes $\beta$-sheets. ${ }^{12-14}$ SDS is also reported to alter the tertiary structure keeping the secondary structure unchanged in some proteins such as cytochrome c. ${ }^{12,15-21}$ It as well affects oligomeric states of various proteins. For instance, $\mathrm{EmrE}^{22}$ from small multidrug resistance (SMR) protein family, BRICHOS ${ }^{23}$ domain trimer (related to human amyloid disease) and multioligomeric haemoglobin $^{24}$ are reported to form either monomers or dissociate in presence of SDS. Similarly suppression of aggregation is observed for insulin ${ }^{25}$ and alcohol dehydrogenase in presence of SDS.

The main objective of the present studies is as follows. The homologues of STY3178 from other virulent bacteria like Salmonella Typhimurium, Escherichia coli, Klebsiella pneumoniae, Salmonella paratyphi yfdx proteins, are all predicted as periplasmic proteins in Cello. ${ }^{26,27}$ The interactions of $\mathrm{yfdX}$ protein with surfactant can possibly mimic the interaction between cell membrane and periplasmic proteins. This leads us to probe the interactions of surfactant with STY3178. Among these yfdX proteins, two are identified as multimers. STY3178 is trimeric in solution as reported in our earlier study. ${ }^{7}$ The homologue of STY3178 from $K$. pneumoniae is shown as 
a tetramer in the deposited structure in PDB. Surfactants at high concentrations are reported to dissociate complex structures of proteins. $^{28-32}$ Moreover, it is also known that proteins get unfolded in presence of high surfactant concentration. ${ }^{28-32}$ The key question is: does STY3178 retain its secondary and tertiary structure in SDS?

Structural changes of STY3178 in presence of SDS are monitored using dynamic light scattering (DLS), size exclusion chromatography (SEC), steady state fluorescence, circular dichroism (CD) and isothermal titration calorimetry (ITC) in our present study. DLS measurements show decrease in hydrodynamic size of the protein with increase in SDS concentration, indicating dissociation of STY3178 trimer which is further confirmed by SEC. We find gradual lowering of fluorescence emission intensity without any effect in the peak position in presence of SDS. Near UV-CD spectrum shows loss of broad shoulder in SDS whereas far-UV CD show the $\alpha$-helical secondary structure remains intact. ITC measurement indicates two regimes during the titration of SDS to the protein, which matches with the DLS observations. To further understand the SDS binding, we perform molecular dynamics simulation. It has been shown that the stabilization of protein-ligand systems can be understood from the conformational free energy and entropy of the system..$^{3,34}$ Our earlier works show that these quantities can be extracted from the distribution of dihedral angles over the simulated trajectory. Further, the destabilized and disordered residues in a given conformation in reference to another, serve as ligand binding sites in proteins for further activities. ${ }^{35,36}$ We estimate the conformational thermodynamics changes and observe the SDS induced stability of modeled monomer of the protein compared to that in the absence of SDS. Our data support our experimental observations of SDS interactions with STY3178.

\section{Methods}

The protocol used for overexpression, extraction and purification of STY3178 is same as reported in our previous study. ${ }^{7}$

\section{Sample preparation}

Sodium dodecyl sulphate (SDS). $5 \mu \mathrm{M}$ protein is incubated overnight in buffers (50 mM potassium phosphate, $\mathrm{pH} 7$, $250 \mathrm{mM} \mathrm{NaCl}$ and $1 \mathrm{mM}$ PMSF) having SDS. Various SDS concentration used during the experiment are $0.696 \mathrm{mM}$ (0.02\%), $1.39 \mathrm{mM}(0.04 \%), 2.1 \mathrm{mM}(0.06 \%), 2.78 \mathrm{mM}(0.08 \%)$, $3.48 \mathrm{mM}(0.1 \%), 8.7 \mathrm{mM}$ (0.25\%), $17.4 \mathrm{mM}$ (0.5\%), $34.8 \mathrm{mM}$ (1\%) and $69.6 \mathrm{mM}(2 \%)$. Samples for background correction are prepared similarly without protein for each experiment.

\section{Dynamic light scattering}

Effect of SDS on hydrodynamic size of the protein is monitored using dynamic light scattering in a Nano-S Malvern instrument at $20{ }^{\circ} \mathrm{C}$. The wavelength of the laser and angle of scattering used for all measurements are $632.8 \mathrm{~nm}$ and $173^{\circ}$, respectively. Protein concentration of $10 \mu \mathrm{M}$ is used, all samples are equilibrated over night and measurement is performed using a10 $\mathrm{mm}$ path length cell. In another set of experiment, $200 \mu \mathrm{M}$ protein is first incubated overnight in $173.5 \mathrm{mM}$ (5\%) SDS. The SDS concentration is then reduced by various folds of dilution keeping the protein concentration fixed at $10 \mu \mathrm{M}$. The measurement for each sample is collected as an average of five scans. The reported data are averaged over two sets of measurement. Error bars represent the standard deviation between the two sets.

\section{Size exclusion chromatography}

We perform SEC for STY3178 in presence of different SDS concentrations using superdex 75 column as per the protocol described earlier. ${ }^{7}$ We subject the protein to SEC in presence of $3.48 \mathrm{mM}(0.1 \%)$ or $69.6 \mathrm{mM}(2 \%)$ SDS. STY3178 $(400 \mu \mathrm{M})$ is first incubated in presence of these SDS concentrations before loading onto the column. The column is pre-equilibrated with buffer having $50 \mathrm{mM}$ phosphate ( $\mathrm{pH} 7), 250 \mathrm{mM} \mathrm{NaCl}, 1 \mathrm{mM}$ PMSF and $3.48 \mathrm{mM}(0.1 \%)$ or $69.6 \mathrm{mM}(2 \%)$ SDS. The protein is eluted at a flow rate of $1 \mathrm{ml}$ per minute using a peristaltic pump (GE Healthcare). Various standard proteins such as lysozyme (14.4 kDa), carbonic anhydrase (29 kDa), ovalbumin (43 kDa), BSA (66 kDa) and conalbumin $(75 \mathrm{kDa})$ are used for column calibration as reported earlier. ${ }^{7}$

\section{Circular dichroism}

The changes in secondary structure for the above protein samples having different concentration of SDS are monitored in Jasco J-815 CD spectrometer. All measurements at room temperature in the far-UV region (200-250 $\mathrm{nm}$ ) are performed using a cuvette of $1 \mathrm{~mm}$ path length and near UV-CD region $(250-330 \mathrm{~nm})$ in $10 \mathrm{~mm}$ cell. Scan speed of $100 \mathrm{~nm} \mathrm{~min} \mathrm{mis}^{-1}$ is used for acquiring the spectra. Data are collected as an average of two scans. Buffer solutions with respective concentration SDS are subtracted from all the data sets.

\section{Intrinsic fluorescence measurements}

The steady state fluorescence emission experiments in presence of various concentration of SDS are recorded in Jobin Yvon Horiba Fluorolog. Protein incubated in buffers with different SDS concentration are excited at 275, 280 and $295 \mathrm{~nm}$ excitation wavelengths using $10 \mathrm{~mm}$ path length quartz cell and $3 \mathrm{~nm}$ slit width. The buffer containing SDS data for background correction are recorded similarly and subtracted from the respective protein spectra.

\section{Isothermal titration calorimetry (ITC)}

We perform ITC measurements using MicroCal iTC200 calorimeter (GE healthcare) to determine the dissociation of STY3178 trimer in presence of SDS at $298 \mathrm{~K}$. STY3178 in a buffer containing $30 \mathrm{mM}$ phosphate (pH 7), $150 \mathrm{mM} \mathrm{NaCl}$ and $1 \mathrm{mM}$ PMSF is titrated against $500 \mathrm{mM}$ SDS loaded in the syringe. Concentration of protein used in the cell is $497 \mu \mathrm{M}$. Reference power used in ITC is $10 \mu \mathrm{cal} \mathrm{s}^{-1}$. First injection of $0.4 \mu \mathrm{l}$ and rest 25 injections of $1.5 \mu \mathrm{l}$ each are injected with $50 \mathrm{rpm}$ constant stirring. Duration of each injection is $3 \mathrm{~s}$ with a time difference 
of $200 \mathrm{~s}$ between two consecutive injections and an initial delay of $60 \mathrm{~s}$ prior to the first injection. The heat of demicellization upon dilution of SDS is subtracted from that of SDS titration into protein. Integrated data is plotted using MicroCal origin.

\section{Docking, simulation and conformational thermodynamics}

STY3178 monomer ${ }^{9}$ is docked with anionic surfactant sodium dodecyl sulphate (SDS) using Autodock $4 .^{37}$ The average structure of the simulated STY3178 monomer is used as a receptor for docking. Residues used for biasing the docking of SDS molecule are N114, R149, Q175, S184 and V185, which are the conformationally destabilized interfacial residues in the trimeric assembly compared to the isolated monomer. ${ }^{8}$ We choose the best docked complex out of five solutions based on minimum energy criterion. The resulting docked complex is minimized and molecular dynamics (MD) simulation is performed in $\mathrm{NAMD}^{38}$ for 40 ns. All-atom molecular dynamics simulations for STY3178 monomer-SDS complex is carried out using the NAMD ${ }^{38}$ program in presence of explicit water molecules (TIP3P model) and counter ions. MD simulations is performed using the CHARMM27 (ref. 39) force field parameters at $310 \mathrm{~K}$ and $1 \mathrm{~atm}$ pressure in isothermal-isobaric (NPT) ensemble, with 1 femto second time step, following standard protocols. ${ }^{33,35,36,40-42}$

We consider the equilibrated ensemble of the docked complex between 10-40 ns for thermodynamic calculations. The conformational thermodynamic changes of SDS bound STY3178 monomer with respect to isolated monomer is calculated using the histogram based method (HBM), ${ }^{33,35,36,40-42}$ the detailed description of which can be found in a recent study. ${ }^{8,33}$ The equilibrated MD trajectory is divided into small time windows (10 ns each) to generate dihedral histograms for every time window. The conformational free energy and entropy have been calculated for each window and averaged over them for each monomer. The error has been estimated from the standard deviations of these values.

\section{Results and discussion}

Native STY3178 protein has a hydrodynamic diameter of about $6.5 \mathrm{~nm}$ in solution as reported earlier. ${ }^{7}$ This hydrodynamic size of the protein is retained even in presence of $8.7 \mathrm{mM}(0.25 \%)$ SDS concentration (Fig. 1A). At $17.4 \mathrm{mM}(0.5 \%)$ SDS, hydrodynamic diameter of the protein decreases to around $6 \mathrm{~nm}$. On further increasing SDS concentration, we observe a gradual decrease in hydrodynamic diameter. At $69.6 \mathrm{mM}$ (2\%) SDS, it decreases further to about $4.55 \mathrm{~nm}$ (Fig. 1A). The reduced hydrodynamic size is similar to proteins like soyabean trypsine inhibitor and carbonic anhydrase with molecular weights 20 $\mathrm{kDa}$ and $29 \mathrm{kDa}$, respectively. ${ }^{7}$ This suggests possibility of STY3178 trimer dissociation into monomer subunits in presence of $69.6 \mathrm{mM}(2 \%)$ SDS. Increasing the SDS concentration further does not change the hydrodynamic size substantially. In presence of $173.5 \mathrm{mM}(5 \%)$ SDS, the hydrodynamic diameter observed is similar to $69.6 \mathrm{mM}(2 \%)$ SDS data within error. Upon reducing SDS concentration from the solution containing
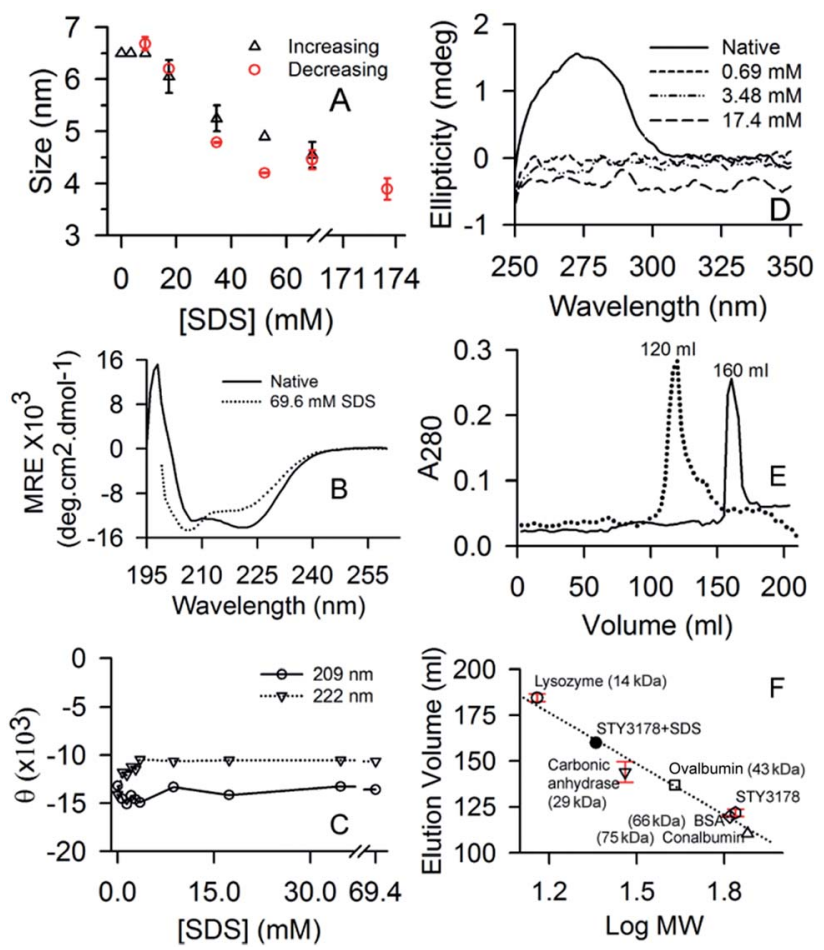

Fig. 1 Effect of SDS on STY3178. (A) Shows the plot of hydrodynamic diameter of protein upon increasing (black) and decreasing (red) SDS concentration. Error bars represent the standard deviation between two sets of measurements. (B) CD spectra of protein in native state (solid) and in presence of $69.6 \mathrm{mM}$ (dotted) SDS. (C) The variation in ellipticity values at $209 \mathrm{~nm}\left(\theta_{209}\right)$ and $222 \mathrm{~nm}\left(\theta_{222}\right)$ wavelengths as a function of SDS concentration are shown using triangle and circle, respectively. (D) Shows the near UV-CD (250-350 nm) spectra of STY3178 in presence of $0.69 \mathrm{mM}$ (short dash), $3.48 \mathrm{mM}$ (dash-dot-dot) and $17.4 \mathrm{mM}$ (long dash) SDS concentration. The native protein spectrum is shown in solid. (E) Shows the elution profile of STY3178 in presence of $3.48 \mathrm{mM}$ (dotted) and $69.6 \mathrm{mM}$ (solid) SDS from superdex 75 column. (F) Represents the calibration curve of superdex 75 column showing the plot of elution volume versus logarithm of molecular weight of various standard proteins lysozyme (14 kDa, circle), carbonic anhydrase (29 kDa, inverted triangle), ovalbumin (45 kDa, square), BSA ( $66 \mathrm{kDa}$, diamond) and conalbumin (75 kDa, triangle). Elution volume of STY3178 without and with 69.6 mM SDS is shown by hexagon and filled circle, respectively.

$173.5 \mathrm{mM}$ (5\%) SDS, we observe increase in hydrodynamic size at $52.2 \mathrm{mM}(1.5 \%)$ SDS concentration (Fig. 1A). The native protein-like hydrodynamic diameter is observed when SDS concentration is reduced to $8.7 \mathrm{mM}(0.25 \%)$. This indicates that around CMC of SDS, STY3178 remains as a trimer. Thus trimer to monomer conversion of STY3178 induced by SDS is reversible in nature.

Far UV-CD spectrum of SY3178 shows its $\alpha$-helical nature as reported earlier ${ }^{7}$ (Fig. 1B). In presence of $69.6 \mathrm{mM}$ SDS (2\%), where we obtain a monomer like hydrodynamic size, the CD spectrum is slightly altered. The two minima at 209 and $222 \mathrm{~nm}$ show a slight variation in ellipticity. However, the spectrum does not look like an unfolded protein CD signature indicating that the conformation of STY3178 in presence of $69.6 \mathrm{mM}$ SDS $(2 \%)$ is folded. When we monitor the ellipticity values at 209 and $222 \mathrm{~nm}$ for increasing concentration of SDS (0-69.6 mM), we 
observe ellipticity decreases at $222 \mathrm{~nm}$ and increases at $209 \mathrm{~nm}$ for $3.48 \mathrm{mM}(0.1 \%)$ SDS. Beyond this concentration of SDS, no substantial change in ellipticity is observed for these two wavelengths (Fig. 1C). Overall there may be a slight change in helix content compared to the native protein keeping the secondary structure similar. Decrease in helix content in presence of $69.6 \mathrm{mM}$ (2\%) SDS compared to native is about $9.3 \%$.

The near UV-CD signature of the protein contains broad shoulder ${ }^{7}$ in the range $250 \mathrm{~nm}$ to $280 \mathrm{~nm}$, which gets altered in presence of $0.696 \mathrm{mM}(0.02 \%)$ SDS (Fig. 1D). This broad shoulder between $250-280 \mathrm{~nm}$ arises due to the presence of phenylalanine and tyrosine in the tertiary structure as reported earlier. ${ }^{7}$ It has been reported earlier that aromatic residues interact with SDS. ${ }^{43}$ This might be the reason due to which the broad shoulder of protein is not observed in the near UV-CD spectrum, even at the lowest concentration of SDS. Similarly in fluorescence emission in presence of SDS, we observe decrease in intensity for the three excitation wavelengths 275 , 280 and $295 \mathrm{~nm}$ (data not shown). There is no change observed in the emission peak position in presence of SDS.

We perform size exclusion chromatography (SEC) using superdex 75 column to confirm the possible dissociation of STY3178 trimer in presence of SDS. SEC is performed in presence of $3.48 \mathrm{mM}(0.1 \%)$ and $69.6 \mathrm{mM}(2 \%)$ SDS and elution volumes are compared with that of the native protein. Fig. $1 \mathrm{E}$ shows the chromatogram of protein eluted from the column. The elution volume of protein in $3.48 \mathrm{mM}$ SDS is around $120 \mathrm{ml}$ and matches with that of the native STY3178 (Fig. 1E). This confirms that STY3178 remains in trimeric state in presence of $3.48 \mathrm{mM}$ SDS. However, the protein in $69.6 \mathrm{mM}$ SDS elutes at a volume of around $160 \mathrm{ml}$ (Fig. 1E). We compare this elution volume with that of several other standard proteins eluted from the same superdex 75 column. The elution volume of $160 \mathrm{ml}$ corresponds to a protein with molecular weight of about $23 \mathrm{kDa}$ (Fig. 1F), confirming SDS-induced dissociation of trimeric STY3178 into its monomers.

We further perform ITC measurements to determine interaction of STY3178 with SDS. Fig. 2A shows the thermogram of titration of SDS into STY3178 solution at $298 \mathrm{~K}$. The corresponding isotherm is shown in Fig. 2B. There are two regions in the ITC isotherm (Fig. 2B). In the first region (up to 100 molar ratio) up to $14^{\text {th }}$ injection, the final SDS concentration is $33.2 \mathrm{mM}(0.95 \%)$ (Fig. 2B). In the same SDS concentration range, we find decrease in hydrodynamic diameter from $6.5 \mathrm{~nm}$ to $5.6 \mathrm{~nm}$ in DLS (Fig. 1A). The second region in ITC isotherm corresponds to SDS concentration of about $63.2 \mathrm{mM}(1.8 \%)$ at final injection (Fig. 2B), where we observe hydrodynamic diameter $(\sim 4.2 \mathrm{~nm})$ of monomeric protein in DLS (Fig. 1A). The ITC data suggest that SDS binds to the trimeric assembly and the corresponding monomer differently.

To further understand qualitatively the SDS binding and stabilization of the monomer in presence of SDS, we perform molecular dynamics (MD) simulation on model system. We consider a monomeric STY3178 and one SDS molecule in our model studies. A couple of points are noteworthy on the construction of our model system: (1) SDS interaction takes place with both monomeric and trimeric protein. For simplicity
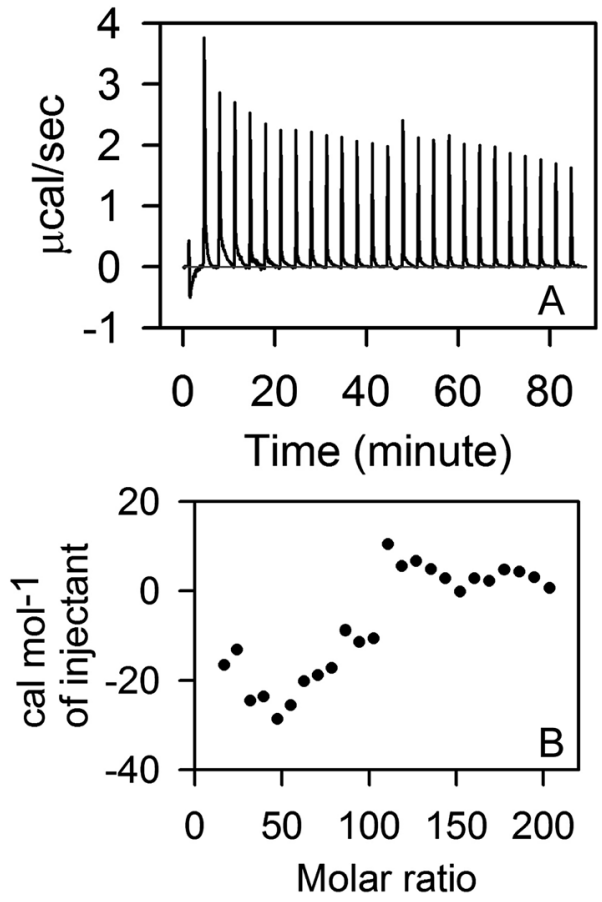

Fig. 2 ITC measurement in presence of SDS. (A) Shows the thermogram of titration of $500 \mathrm{mM}$ SDS into STY3178. (B) Shows the isotherm of SDS binding with STY3178.

of the calculation we restrict only to the monomeric state. (2) We have considered one SDS molecule, even though the experiments indicate that the protein monomers are stable for large SDS concentrations much above the CMC where the SDS molecules form micelles. The SDS molecules are amphiphilic in nature, containing negatively charged head-group and hydrophobic tail. Due to amphiphilic nature, even in the micelles the head groups are solvent exposed which can interact with protein as in a single SDS molecule. Thus the interactions responsible for SDS and the protein interactions have been adequately taken into account in our model calculations.

We have shown earlier a simulated monomer model structure which can explain experimentally observed thermal stability. ${ }^{9}$ In a recent study we have shown the MD simulated trimeric conformations of STY3178 and compared its stability over the monomer structure. ${ }^{8}$ We observe that the trimeric conformation is more stable compared to the monomer. However, there are some interfacial residues in the trimer which are destabilized with respect to the monomer. ${ }^{8}$ The changes in conformational free energy, $\Delta G_{\mathrm{i}}^{\text {conf }}$ and entropy, $T \Delta S_{\mathrm{i}}^{\text {conf }}$ of these destabilized interfacial residues between two monomers are shown in Table 1 where the data is averaged over all the three chains. $\Delta G_{\mathrm{i}}^{\text {conf }}$ and $T \Delta S_{\mathrm{i}}^{\text {conf }}$ in the table are quoted in units of $\mathrm{kJ} \mathrm{Mol}^{-1}$. The estimated error for $\Delta G_{\mathrm{i}}^{\text {conf }}$ is approximately $4 \%$ and that for $T \Delta S_{\mathrm{i}}^{\text {conf }}$ is $5 \%$. Within this error bar the conformational free energy of the lowest magnitude still remains positive $(0.02 \pm 0.0008)$ indicating destabilization, albeit with very small magnitude. The total destabilization conformational free energy of the relevant residues add up to about $1 k_{\mathrm{B}} T$. 
Table 1 Conformational thermodynamic changes (in units of $\mathrm{kJ}$ $\mathrm{mol}^{-1}$ ) of destabilized interfacial residues in the trimeric assembly

\begin{tabular}{llc}
\hline $\begin{array}{l}\text { Interfacial } \\
\text { residues }\end{array}$ & $\Delta G_{\mathrm{i}}^{\text {conf }}$ & $T \Delta S_{\mathrm{i}}^{\text {conf }}$ \\
\hline $\mathrm{N} 114$ & 0.1 & -0.5 \\
$\mathrm{R} 149$ & 0.02 & -2.3 \\
$\mathrm{Q} 175$ & 0.06 & -0.02 \\
$\mathrm{~S} 184$ & 0.8 & 0.6 \\
V185 & 0.7 & 1.2 \\
E187 & 0.3 & 0.3
\end{tabular}

The destabilized and disordered polar, basic or hydrophobic residues can bind to amphiphilic SDS molecule. If we consider the destabilized and disordered residues as shown in Table 1, we observe that the majority of the residues are polar, along with basic and hydrophobic ones as well. The destabilized and disordered polar, basic or hydrophobic residues can bind amphiphilic SDS molecule with negatively charged head group. This is because of the amphiphilic nature of SDS containing negatively charged head-group and hydrophobic tail. If we consider the destabilized and disordered residues as shown in Table 1 , we observe $66 \%$ of them are polar, $17 \%$ is basic and $17 \%$ is hydrophobic.

We perform biased docking of SDS molecule with a simulated monomer of STY3178 using N114, R149, Q175, S184 and V185 as active residues. The docked monomer-SDS complex is then simulated to obtain ensemble of structures. We estimate conformational thermodynamic changes of SDS bound monomer with respect to SDS-free monomer. The simulated docked complex is shown in Fig. 3A. The SDS molecule after simulation stabilizes near the residues V180, S182, Q183, S184, V185, Q186 and S188, which forms the binding region. The SDS bound monomer shows overall gain in conformational stability, $\Delta G_{\text {conf }}$ $=-100.3 \mathrm{~kJ} \mathrm{~mol}^{-1}$ and ordering $T \Delta S_{\text {conf }}=-431.8 \mathrm{~kJ} \mathrm{~mol}^{-1}$ with respect to an isolated monomer. A residue wise conformational thermodynamics analysis upon SDS binding show that in the SDS-monomer complex, residues V180, S182, Q183, S184, V185, Q186 and S188 get stabilized. The conformational free energy changes, $\Delta G_{\mathrm{i}}^{\text {conf }}$, shown in black, in Fig. 3B, are computed for monomer-SDS complex with respect to the free monomer. We compare the $\Delta G_{\mathrm{i}}^{\text {conf }}$ (grey) for these residues in the trimeric assembly, where we compute conformational free energy changes of the trimer with respect to monomer. The residuewise conformational free energy changes in the trimer is computed by taking an average over the three units (Fig. 3B). $\Delta G_{\mathrm{i}}^{\text {conf }}$ of V180, Q186 and S188 are negative in both the trimeric assembly as well as in the monomer-SDS complex, albeit the degree of stabilization of Q186 is more upon SDS binding. The other residues, S182, Q183, S184 and V185, which are destabilized $\left(\Delta G_{\mathrm{i}}^{\text {conf }}>0\right)$ in the trimeric assembly, undergo conformational stabilization upon SDS binding.

The simulation results indicate that SDS binding stabilizes monomeric STY3178. This is in qualitative agreement to our experimental observations that high concentration of SDS dissociates trimeric STY3178 and stabilizes the monomer.
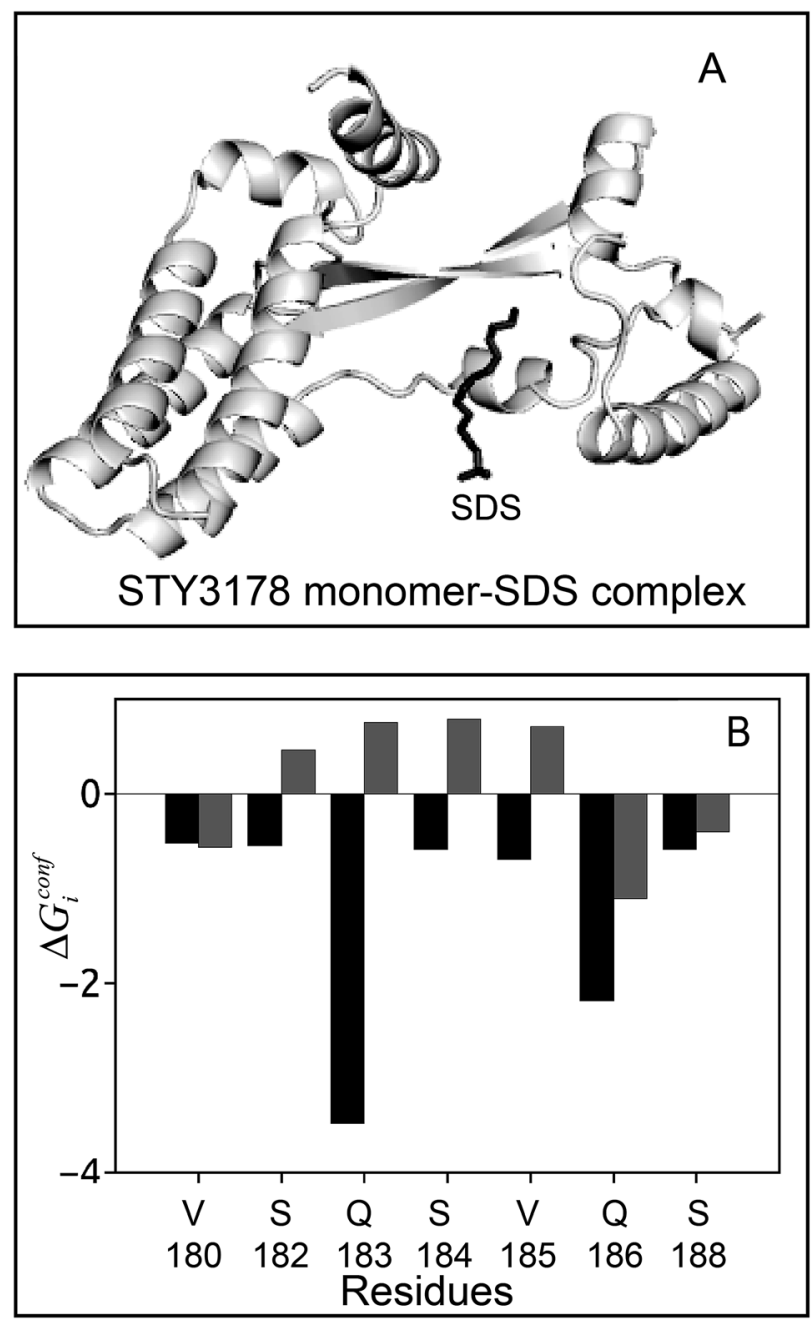

Fig. 3 STY3178-SDS docked structure and free energy change (in units of $\mathrm{kJ} \mathrm{mol}^{-1}$ ). (A) Final simulated structure of STY3178 monomerSDS complex. (B) The conformational free energy changes, $\Delta G_{i}^{\text {conf }}$ of SDS binding residues in monomer-SDS complex (black). The $\Delta G_{i}^{\text {conf }}$ of same residues in the trimer assembly (grey) calculated as an average over three monomers. The thermodynamic calculations are performed with respect to an isolated monomer.

These observations are similar to the interactions between surfactants and multimeric proteins reported earlier. ${ }^{28-32} \mathrm{We}$ also show that monomer-monomer interface of the trimeric assembly is suitable binding surface for SDS. Since the exposed charged head groups are more in number in SDS micelles, the electrostatic interactions with the destabilized polar residues will be enhanced compared to the single SDS molecules. This may lead to difference between the binding of SDS with STY3178 for high and low concentrations of SDS.

\section{Conclusion}

Anionic surfactant SDS at high concentration induces slight secondary structural changes and major tertiary or quaternary changes in STY3178. Trimeric assembly of STY3178 dissociates reversibly in presence of $69.6 \mathrm{mM}(2 \%)$ or higher concentration 
of SDS. However, no SDS induced unfolding of this protein is observed. Our experimental results are substantiated by docking and simulation studies. We observe SDS molecule binds polar and hydrophobic residues present in monomer-monomer interface of the trimer. SDS upon binding the monomeric protein surface induces conformational stability over isolated monomer. These results also indicate that in the vicinity of cell membrane environment as mimic by SDS, STY3178 or other multimeric yfdX proteins might exist as a monomer.

\section{Acknowledgements}

M. G., P. S. and C. M. thank the DBT-CU-IPLS facility for ITC and Souvik Roy for his assistance during ITC measurement. S. S. thanks the UGC for funding. M. G. and J. C. thank the DST for funding. We thank Sutapa Dutta for help in preparing the manuscript.

\section{References}

1 M. Aivaliotis, P. Samolis, E. Neofotistou, H. Remigy, A. K. Rizos and G. Tsiotis, Biochim. Biophys. Acta, 2003, 1615, 69-76.

2 A. Helenius and K. Simons, Biochim. Biophys. Acta, 1975, 415, 29-79.

3 M. M. Nielsen, K. K. Andersen, P. Westh and D. E. Otzen, Biophys. J., 2007, 92, 3674-3685.

4 X. F. Wei, Z. D. Chang and H. Z. Liu, J. Surfactants Deterg., 2003, 6, 107-112.

5 H. Z. Liu, W. J. Yang and J. Y. Chen, Biochem. Eng. J., 1998, 2, 187-196.

6 X. F. Wei and H. Z. Liu, J. Surfactants Deterg., 2000, 3, 491495.

7 P. Saha, C. Manna, S. Das and M. Ghosh, Sci. Rep., 2016, 6, 21305.

8 P. Saha, S. Sikdar, J. Chakrabarti and M. Ghosh, RSC Adv., 2016, 6, 91256-91264.

9 P. Saha, C. Manna, J. Chakrabarti and M. Ghosh, Sci. Rep., 2016, 6, 29541.

10 S. Mammi and E. Peggion, Biochemistry, 1990, 29, 52655269.

11 J. Rizo, F. J. Blanco, B. Kobe, M. D. Bruch and L. M. Gierasch, Biochemistry, 1993, 32, 4881-4894.

12 Q. Xu and T. A. Keiderling, Protein Sci., 2004, 13, 2949-2959.

13 L. Zhong and W. C. Johnson Jr, Proc. Natl. Acad. Sci. U. S. A., 1992, 89, 4462-4465.

14 D. V. Waterhous and W. C. Johnson Jr, Biochemistry, 1994, 33, 2121-2128.

15 K. Hiramatsu and J. T. Yang, Biochim. Biophys. Acta, 1983, 743, 106-114.

16 E. L. Gelamo and M. Tabak, Spectrochim. Acta, Part A, 2000, 56, 2255-2271.

17 E. L. Gelamo, C. H. Silva, H. Imasato and M. Tabak, Biochim. Biophys. Acta, 2002, 1594, 84-99.

18 L. Gebicka and J. L. Gebicki, J. Protein Chem., 1999, 18, 165172.

19 T. K. Das, S. Mazumdar and S. Mitra, Eur. J. Biochem., 1998, 254, 662-670.
20 A. Muga, H. H. Mantsch and W. K. Surewicz, Biochemistry, 1991, 30, 7219-7224.

21 K. Takeda, K. Takahashi and P. P. Batra, Arch. Biochem. Biophys., 1985, 236, 411-417.

22 D. C. Bay, R. A. Budiman, M. P. Nieh and R. J. Turner, Biochim. Biophys. Acta, 2010, 1798, 526-535.

23 H. Biverstal, L. Dolfe, E. Hermansson, A. Leppert, M. Reifenrath, B. Winblad, J. Presto and J. Johansson, Biochim. Biophys. Acta, 2015, 1854, 835-843.

24 E. L. Gelamo, R. Itri and M. Tabak, J. Biol. Chem., 2004, 279, 33298-33305.

25 Y. M. Kwon, M. Baudys, K. Knutson and S. W. Kim, Pharm. Res., 2001, 18, 1754-1759.

26 C. S. Yu, C. J. Lin and J. K. Hwang, Protein Sci., 2004, 13, 1402-1406.

27 C. S. Yu, Y. C. Chen, C. H. Lu and J. K. Hwang, Proteins: Struct., Funct., Bioinf., 2006, 64, 643-651.

28 K. Gimpl, J. Klement and S. Keller, Biol. Proced. Online, 2016, $18,4$.

29 G. G. Prive, Methods, 2007, 41, 388-397.

30 C. Breyton, C. Tribet, J. Olive, J. P. Dubacq and J. L. Popot, J. Biol. Chem., 1997, 272, 21892-21900.

31 B. Mandal, S. Ghosh and S. P. Moulik, New J. Chem., 2016, 40, 4617-4624.

32 U. Anand and S. Mukherjee, Phys. Chem. Chem. Phys., 2013, 15, 9375-9383.

33 A. Das, J. Chakrabarti and M. Ghosh, Biophys. J., 2013, 104, 1274-1284.

34 K. K. Frederick, M. S. Marlow, K. G. Valentine and A. J. Wand, Nature, 2007, 448, 325-329.

35 S. Sikdar, J. Chakrabarti and M. Ghosh, Mol. BioSyst., 2014, 10, 3280-3289.

36 S. Sikdar, J. Chakrabarti and M. Ghosh, Mol. BioSyst., 2016, 12, 444-453.

37 G. M. Morris, R. Huey, W. Lindstrom, M. F. Sanner, R. K. Belew, D. S. Goodsell and A. J. Olson, J. Comput. Chem., 2009, 30, 2785-2791.

38 J. C. Phillips, R. Braun, W. Wang, J. Gumbart, E. Tajkhorshid, E. Villa, C. Chipot, R. D. Skeel, L. Kale and K. Schulten, J. Comput. Chem., 2005, 26, 1781-1802.

39 B. R. Brooks, C. L. Brooks 3rd, A. D. Mackerell Jr, L. Nilsson, R. J. Petrella, B. Roux, Y. Won, G. Archontis, C. Bartels, S. Boresch, A. Caflisch, L. Caves, Q. Cui, A. R. Dinner, M. Feig, S. Fischer, J. Gao, M. Hodoscek, W. Im, K. Kuczera, T. Lazaridis, J. Ma, V. Ovchinnikov, E. Paci, R. W. Pastor, C. B. Post, J. Z. Pu, M. Schaefer, B. Tidor, R. M. Venable, H. L. Woodcock, X. Wu, W. Yang, D. M. York and M. Karplus, J. Comput. Chem., 2009, 30, 1545-1614.

40 A. Das, S. Sikdar, M. Ghosh and J. Chakrabarti, J. Phys.: Conf. Ser., 2015, 638, 012013.

41 A. Das, J. Chakrabarti and M. Ghosh, Chem. Phys. Lett., 2013, 581, 91-95.

42 A. Das, J. Chakrabarti and M. Ghosh, Mol. BioSyst., 2014, 10, 437-445.

43 S. M. Kelly, T. J. Jess and N. C. Price, BBA Proteins and Proteomics, 2005, 1751, 119-139. 